\title{
SÍNDROME DE GILLES DE LA TOURETTE
}

\section{Estudo clínico de 58 casos}

\author{
Chien Hsin Fen ${ }^{1}$, Egberto Reis Barbosa ${ }^{2}$, Eurípedes Constantino Migue $\beta$
}

\begin{abstract}
RESUMO - A síndrome de Gilles de la Tourette (SGT) é um transtorno neuropsiquiátrico, de início na infância, caracterizado pela presença de tiques motores e vocais. Cinquenta e oito pacientes com SGT foram acompanhados clinicamente durante período de três anos. Trinta e seis pacientes eram do sexo masculino e 22 do feminino, a proporção entre os sexos foi 1,6:1. A idade dos pacientes variou de 7 a 50 anos, média 20,33 anos. A idade de início dos sintomas variou de 3 a 15 anos, sendo a média 7,81 anos. 0 tique motor foi o sintoma inaugural em $79 \%$ dos pacientes. Quanto aos tiques complexos, a coprolalia estava presente em 27,6\%; a copropraxia em 20,1\%; a palilalia em 20,1\%; a ecolalia em 27,6\%; e a ecopraxia em $27,6 \%$. Em relação a manifestações associadas, 25,8\% apresentavam déficit de atenção/ hiperatividade e 39,6\% transtorno obsessivo-compulsivo. 0 fenômeno sensitivo estava presente em $54,8 \%$ dos pacientes.
\end{abstract}

PALAVRAS-CHAVE: síndrome de Gilles de la Tourette, tiques.

Gilles de la Tourette syndrome: a clinical study on 58 cases

ABSTRACT - Gilles de la Tourette's syndrome (GTS) is a neuropsychiatric disorder with a childhood onset and is characterized by motor and vocal tics. Fifty-eight patients with GTS were evaluated during a period of three years. Thirty-six patients were male and twenty two female, with an age range of 7 to 51 years (mean 20.33 years). The male to female ratio was 1.6:1. The age of onset ranged from 3 to 15 years (mean 7.81 years). Seventy nine percent of the patients presented motor tics as the initial symptom of the disease. In terms of complex tics, coprolalia was present in $27.6 \%$ of the patients; copropraxia in $20.1 \%$; palilalia in $20.1 \%$; ecolalia in $27.6 \%$; and ecopraxia in 27,6\%. Associated manifestations, such as attention deficit, hyperactivity disorder and obsessive-compulsive disorder were present in $25.8 \%$ and $39.6 \%$, respectively. Sensory phenomena were present in $54.8 \%$ of the patients.

KEY WORDS: Gilles de la Tourette's syndrome, tics.

A síndrome de Gilles de la Tourette (SGT) é transtorno neuropsiquátrico de início na infância, que se caracteriza pela presença de tiques motores e vocais $^{1,2}$. Desde a sua primeira descrição $0^{3,4} \mathrm{em} 1885$, muito aspectos dessa entidade nosológica ainda permanecem obscuros. Parte desse desconhecimento pode ser atribuído ao fato de que, até há poucas décadas, muitos profissionais da área de saúde desconheciam as características clínicas ou não admitiam causa orgânica para este transtorno ${ }^{5}$.

A partir dos anos 60, pesquisadores em todo o mundo vêm descrevendo novos aspectos desse transtorno ${ }^{6,7}$. Sabe-se hoje que essa condição está associada a alterações neurofisiológicas e neuroanatômicas ${ }^{8}$ de etiologia, no entanto, ainda desconhecida. Há ainda inúmeras lacunas a serem esclarecidas tais como: um modelo neurobiológico preciso, o modo de transmissão genética e o espectro clínico desse transtorno.

Descreveremos os aspectos clínicos de 58 pacientes com SGT acompanhados no Ambulatório de Distúrbios do Movimento do Departamento de Neurologia do HC-FMUSP'.

\section{MÉTODO}

Foram analisados os dados de 58 pacientes com diagnóstico de SGT atendidos no Ambulatório de Distúrbios do Movimento da Clínica Neurológica do HC-FMUSP. Para

Divisão de Clínica Neurológica do Hospital das Clínicas (HC) da Faculdade de Medicina da Universidade de São Paulo (FMUSP), São Paulo SP, Brasil: ${ }^{1}$ Mestre em Neurologia FMUSP; ${ }^{2}$ Professor Livre-Docente de Clínica Neurológica, HC-FMUSP; ${ }^{3}$ Professor Assistente do Departamento de Psiquiatria, FMUSP. Este estudo foi realizado com o apoio do Conselho Nacional de desenvolvimento científico e Tecnológico (CNPq)

Recebido 30 Janeiro 2001, recebido na forma final 16 Maio 2001. Aceito 23 Maio 2001.

Dr. Egberto Reis Barbosa - Clínica Neurológica, Hospital das Clínicas, FMUSP - Av. Dr. Enéas de Carvalho Aguiar 255 - $05403-000$ - São Paulo SP-Brasil. 


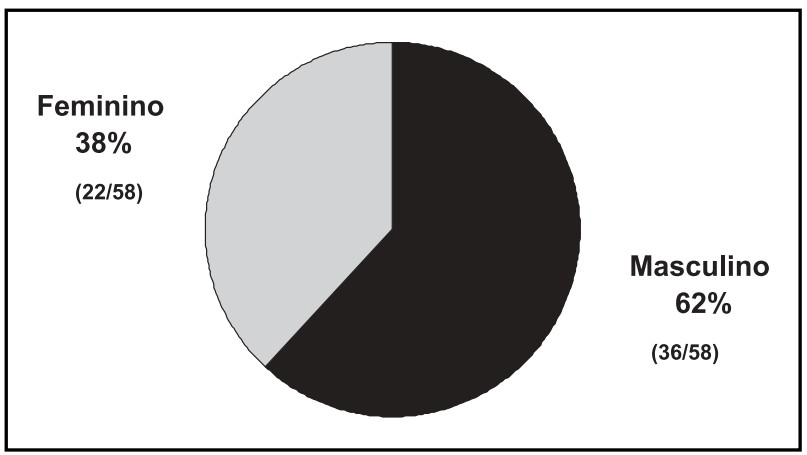

Fig 1. Distribuição quanto ao sexo.

o diagnóstico de SGT utilizamos os critérios clínicos do Tourette Syndrome Classification Study Group ${ }^{9}$. Para o diagnóstico de transtorno de déficit de atenção sem/com hiperatividade (TDAH) e transtorno obsessivo-compulsivo (TOC), utilizamos os critérios do DSM-IV ${ }^{10}$.

Dos 58 pacientes, em 42 os dados foram obtidos diretamente durante o período de Fevereiro de 1996 a Março de 1999 e nos 16 restantes, por meio da consulta a prontuários.

Foram registrados os seguintes dados de cada paciente: idade de início, idade atual, sexo, manifestações iniciais, presença de tiques complexos, presença de manifestações associadas (TDAH e TOC) e presença de fenômeno sensorial. Este último é referido como uma vivência subjetiva (ou percepção), de definição imprecisa na literatura. O Tourette Syndrome Classification Study Group ${ }^{9}$ definiu o fenômeno sensorial como sentimentos generalizados ou focais que precedem os tiques e geralmente são aliviados com o movimento. Existem ainda outros conceitos para este termo de acordo com a revisão de Miguel et $\mathrm{al}^{26}$.

Todos os pacientes foram examinados sob o ponto de vista neurológico e não foi encontrada nenhuma alteração além dos tiques motores ou vocais. O exame de tomografia computadorizada do encéfalo foi realizado em todos os pacientes e nenhuma alteração significativa foi evidenciada.

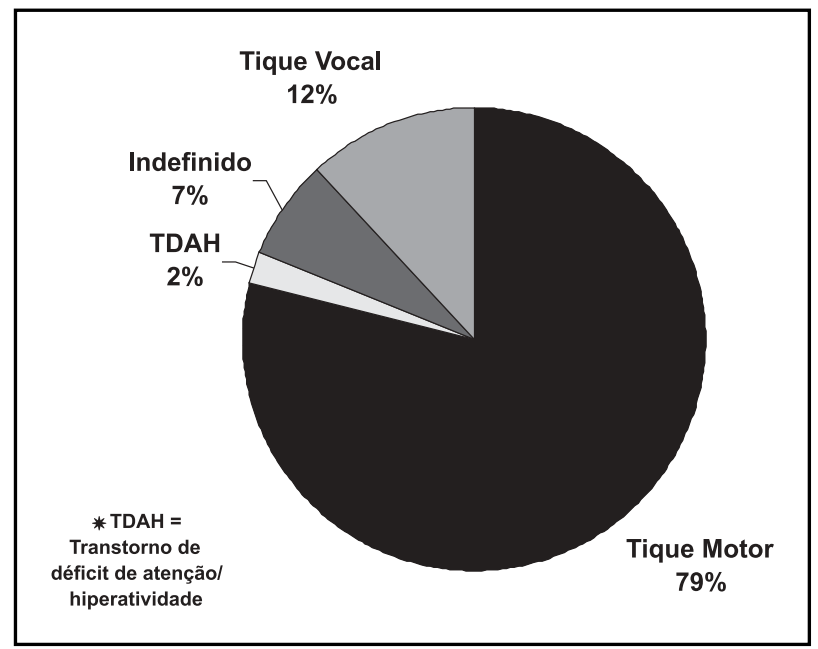

Fig 2. Manifestações inaugurais da Síndrome de Gilles de la Tourette.

\section{RESULTADOS}

Dos 58 pacientes estudados, 22 (38\%) eram do sexo feminino e 36 (62\%) do masculino. A proporção entre pacientes do sexo masculino e feminino era 1,6:1 (Fig 1).

A idade atual dos pacientes variou de 7 a 50 anos, sendo a média 20,33 anos e a mediana 17 anos. A idade de início das manifestações clínicas variou de 3 a 15 anos, com média de 7,83 anos e mediana de 7 anos.

Em 46 (79\%) pacientes, a manifestação inaugural da SGT foi o tique motor. O tique vocal e o TDAH foram as manifestações inaugurais em 7 (12\%) e 1 (2\%) pacientes, respectivamente. Não foi possível determinar a manifestação inicial em 4 (7\%) casos, porque já apresentavam longo tempo de evolução e não

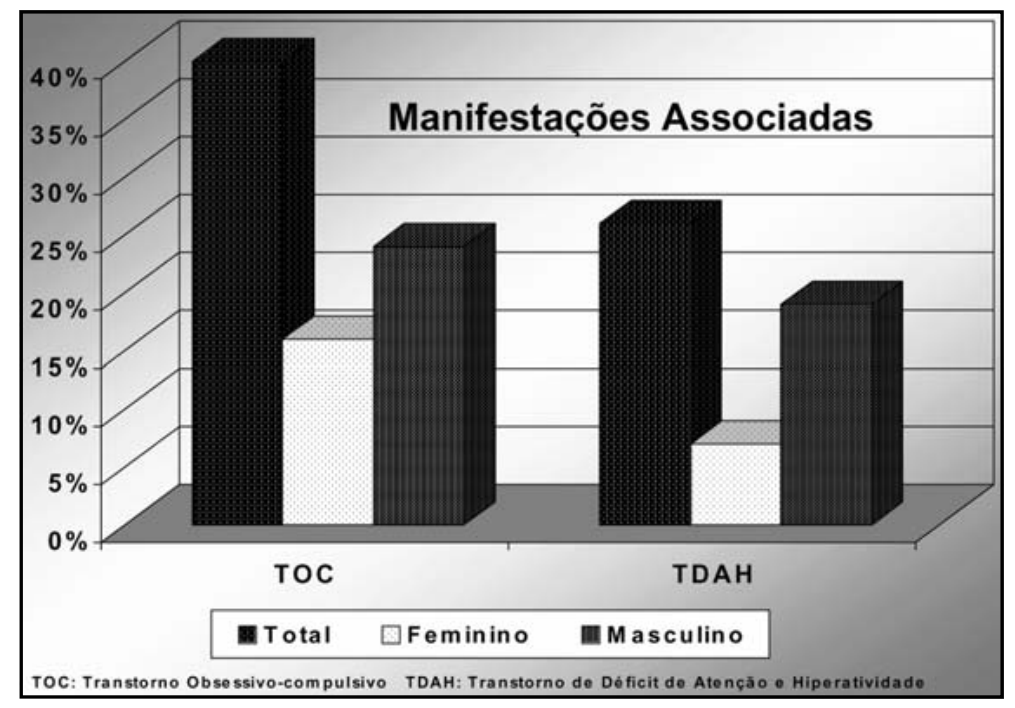

Fig 3. Frequência de manifestaçôes associadas e sua distribuição quanto ao sexo. 
se lembravam de detalhes do início do quadro. Os dados referentes às manifestações inaugurais da doença constam na Figura 2.

Quanto aos tiques complexos, 16 (27,6\%) pacientes apresentaram coprolalia; $12(20,1 \%)$ copropraxia; $12(20,1 \%)$ palilalia; $16(27,6 \%)$ ecolalia e $16(27,6 \%)$ ecopraxia.

Quanto às manifestações associadas, o TDAH foi observado em $25,8 \%$ (15/58) e o TOC em $39,6 \%$ (23/ $58)$ dos casos estudados. Essas duas condições predominavam em pacientes do sexo masculino (11 em 15 com TDAH e 14 em 23 com TOC), conforme pode observado na Figura 3. Fenômenos sensoriais estavam presentes em 54,76\% (23/42) dos casos.

Quando inquiridos sobre a presença de tiques ou TOC entre os familiares, $24 \%$ dos pacientes (10/42) relataram a presença de tique e/ou TOC em familiares de primeiro grau; $21 \%$ (9/42) em familiares de segundo grau e $5 \%$ (2/42) em familiares de terceiro grau.

\section{DISCUSSÃO}

Apesar de não terem sido encontradas diferenças significativas quanto às manifestações sintomatológicas dos pacientes com SGT neste grupo em comparação com dados da literatura, podem ser caracterizados alguns aspectos particulares nesta série.

Uma característica peculiar do grupo estudado é a predominância de adultos entre os casos avaliados (idade média de 20,33 anos). A análise da distribuição por sexo dos pacientes mostra relação de 1,6 pacientes do sexo masculino para um do sexo feminino, portanto abaixo da encontrada na maioria das casuísticas relatadas na literatura ${ }^{2,11-15}$. Porém, essa proporção é semelhante à encontrada por Apter et al. ${ }^{16}$, que estudaram jovens de 18 anos, recrutados para o serviço militar obrigatório em Israel. Kerbeshian et al. ${ }^{17}$ encontraram uma relação de 9 pacientes do sexo masculino para 1 do feminino com SGT em estudo epidemiológico com crianças de idade escolar e uma relação de 3,4:1 em pacientes de idade adulta da mesma comunidade. Esses dados indicam que há uma diferença de características entre a população adulta e infantil entre os portadores da SGT.

Apter et $\mathrm{al}^{16}$ acreditam que essas diferenças de proporção entre os sexos se deva ao fato de haver para os pacientes do sexo masculino, maior chance de remissão dos sintomas na idade adulta do que para os do sexo feminino.

Brunn e Budman ${ }^{2}$ relatam que cerca de $2 / 3$ dos pacientes têm melhora importante dos tiques na fase adulta. Pelo fato de nosso serviço ser de referência terciária, é possível que concentre maior número de casos com persistência da sintomatologia até a idade adulta (casos de maior gravidade), entre os quais predominam pacientes de sexo feminino.

Quanto ao transtorno obsessivo compulsivo, notamos predominância dos sintomas em pacientes do sexo masculino (14/23). Até há pouco, acreditava-se que o TOC predominava em indivíduos do sexo feminino ${ }^{18}$; entretanto, estudos mais recentes mostram que, quando o paciente apresenta SGT e TOC, há predominância no sexo masculino ${ }^{19-21}$, portanto compatível com os dados encontrados no presente estudo.

Muitos autores relatam que, frequentemente, o TDAH é a primeira manifestação da $\mathrm{SGT}^{22}$. Na presente série, porém, apenas um paciente apresentou TADH $(1,72 \%)$ como manifestação inicial da doença. Uma explicação para esse fato pode ser a faixa etária do grupo em questão, pois tratando-se de adultos, as informações referentes às fases mais precoces da vida quando instalaram-se as primeiras manifestações do transtorno geralmente são mais precárias. Muitas vezes, mesmo os pais não sabem referir se o filho(a) apresentou sintomas de TDAH, pois a instalação dos tiques é mais marcante.

Muitos pacientes referem a presença de fenômenos sensoriais que impelem o paciente a realizar o tique com o intuito de aliviar esse desconforto. Autores como Bliss ${ }^{23}$ consideram os tiques em geral como uma resposta voluntária a esses fenômenos premonitórios. Esse tipo de fenômeno estava presente em $54,76 \%$ dos casos avaliados neste estudo.

Em 1988, Shapiro et al. ${ }^{24}$, em estudo retrospectivo, analisaram a presença de sensação premonitória nos seus 1237 pacientes e encontraram uma freqüência de 8,5\% (105/1237). Shapiro introduziu o termo "tique sensitivo" para esse fenômeno, pois apesar de expressar-se antes dos tiques motores ou vocais, muitas vezes os pacientes referiam a presença dessa sensação independentemente dos tiques. A crítica a esse trabalho recai sobre seu caráter retrospectivo, pois muitas vezes o paciente não refere essa sensação, a não ser quando especificamente inquirido.

Segundo Leckman et al. ${ }^{25}$, quanto maior a idade maior é a percepção do fenômeno sensorial. Sendo esta amostra constituída predominantemente de adultos, deveríamos encontrar porcentagem maior de pacientes com esse tipo de manifestação. Como isso não ocorreu, podemos aventar a hipótese de 
que a frequência do fenômeno sensorial não esteja relacionada com a idade e, portanto, com a capacidade de exprimir adequadamente os sintomas.

Miguel et al. ${ }^{26,27}$, em estudo recente, consideram que a presença de fenômeno sensorial é um dado importante para caracterizar subgrupos no espectro TOC - SGT. Assim, os autores constataram que esse fenômeno é mais frequente em pacientes com TOC associado à SGT do que em pacientes com TOC primário na ausência de tiques.

A média de idade de início da doença observada nesta série foi 7,8 anos, cifra que não difere da constatada em outros estudos, incluindo-se o de Cardoso et al ${ }^{28}$ (32 pacientes avaliados) e o de Alves ${ }^{28}$ (42 pacientes avaliados), ambos desenvolvidos em nosso meio e que registraram idades médias de início de 7,1 anos e 6,8 anos, respectivamente.

Quanto aos tiques complexos, deve-se ressaltar que a coprolalia, manifestação típica mas não obrigatória da SGT, estava presente em apenas $27,6 \%$ dos casos. Na maioria das séries publicadas, esse tipo de manifestação é encontrado em menos de $40 \%$ dos casos; portanto sua ausência não deve trazer dúvidas quanto ao diagnóstico, desde que os critérios essenciais sejam preenchidos.

A significativa freqüência de familiares que apresentavam tique ou TOC está de acordo com a literatura e é indicativa da natureza genética dessa condição. Freeman et al. ${ }^{11}$, em recente estudo multicêntrico envolvendo 22 países e agrupando 3500 casos da SGT (parte dos casos deste estudo integram essa gigantesca série), observaram que, quanto mais precoce a instalação dessa afecção, maior era a positividade de história familiar para tiques.

\section{REFERÊNCIA}

1. Chien HF. Síndrome de Gilles de la Tourette: aspectos clínicos, etiopatogênicos e terapêuticos. Dissertação de Mestrado, Faculdade de Medicina da Universidade de São Paulo. São Paulo, 1999.

2. Brunn RD, Budman CL. The natural history of Tourette syndrome. Adv Neurol 1992;58:1-6.

3. Gilles de la Tourette GAB. Étude sur une affection nerveuse caractérisée par de l'incoordination motrice accompagnée d'écholalie et coprolalie. (Jumping, Latah, Myriachit). Arch Neurol 1885;9:19-42.

4. Gilles de la Tourette GAB. Étude sur une affection nerveuse caractérisée par de l'incoordination motrice accompagnée d'écholalie et coprolalie. (Jumping, Latah, Myriachit). Arch Neurol 1885;9:155-200.
5. Kushner HI. Medical fictions: the case of the cursing marquise and the (re)construction of Gilles de la Tourette's syndrome. Bull Hist Med 1995;69:224-254

6. Shapiro AK, Shapiro E. Treatment of Gilles de la Tourette's syndrome with haloperidol. Br J Psychiatry 1968;114:345-350.

7. Shapiro AK, Shapiro E. Tourette syndrome: history and present status. In Friedhof AJ, Chase T (eds) Gilles de la Tourette syndrome. New York: Raven Press, 1982:17-23.

8. Singer HS. Neurobiology of Tourette syndrome. In Jankovic J (ed) Neurologic clinics. Philadelphia: Saunders, 1997;15:357-379.

9. The Tourette Syndrome Classification Study Group. Definitions and classification of tic disorders. Arch Neurol 1993;50:1013-1016.

10. American Psychiatry Association. Diagnostic and statistical manual of mental disorders. 4.Ed. Washington, DC: APA Press, 1994.

11. Freeman RD, Fast DK, Burd 1, Kebershian J, Robertson MM, Sandor P. An international perspective on Tourette syndrome: selected findings from 3500 individuals in 22 countries. Dev Med Child Neurol 2000;42:436-447.

12. Singer HS, Walkup, JT. Tourette syndrome and other tic disorders: diagnosis, pathophysiology, and treatment. Medicine 1991;70:15-32.

13. Leckman JF, Peterson BS, Pauls DL, Cohen DJ. Tic disorders. In Miguel EC, Rauch SL, Leckman JF (eds). Psychiatry Clinics of North America. Philadelphia: Saunders, 1997;20:839-861.

14. Robertson MM. The Gilles de la Tourette syndrome: the current status. Br J Psychiatry 1989;154:147-169.

15. Robertson MM. Annotation: Gilles de la Tourette syndrome-an update. J Child Psychiatry 1994;35,597-611.

16. Apter A, Pauls DL, Bleich A, et al. A population-based epidemiological study of Tourette syndrome among adolescents in Israel. Adv Neurol 1992;58:61-64.

17. Kerbeshian J, Burd L. The North Dakota prevalence studies of Tourette syndrome and other developmental disorders. Adv Neurol 1992; 58: 67-74.

18. Robertson MM, Yakeley J. Gilles de la Tourette syndrome and obsessivecompulsive disorder. In Fogel BS, Schiffer RB, Rao SM (eds). Neuropsychiatry. Baltimore: William \& Wilkins, 1996:827-870.

19. Leonard HL, Swedo SE, Rapoport JL, et al. Tourette syndrome and obsessive-compulsive disorder. Adv Neurol 1992;58:83-93.

20. George MS, Trimble MR, Ring HA, Sallee FR, Robertson MM. Obsessions in obsessive-compulsive disorder with and without Gilles e la Tourette's syndrome. Am J Psychiatry 1993;150:93-97.

21. Zohar AH, Pauls DL, Ratzoni G, et al. Obsessive-compulsive disorder with and without tics in a epidemiological sample of adolescents. Am J Psychiatry 1997;154:274-276.

22. Jankovic J. Tics and Tourette syndrome. In: Brekfast Seminar 2.8: Tourette Syndrome, The $5^{\text {th }}$ International Congress Of Parkinson's Disease and Movement Disorder. New York: Mov Disord Society, 1998;1-36.

23. Bliss J. Sensory experiences of Gilles de la Tourette syndrome. Arch Gen Psychiatry 1980;37:1343-1347.

24. Shapiro AK, Shapiro ES, Young JG, Feinberg TE. Sensory tics. In Shapiro AK, Shapiro ES, Young JG, Feinberg TE (eds). Gilles de la Tourette syndrome. New York: Raven, 1988;356-360.

25. Leckman JF, Walker DE, Goodman WK, Pauls DL, Cohen DJ. Just right perceptions associated with compulsive behavior in Tourette's syndrome. Am J Psychiatry 1994;151:675-680.

26. Miguel EC, Rosário-Campos MC, Prado HS, et al. Sensory phenomena in obsessive-compulsive disorder and Tourette's disorder. J Clin Psychiatry 2000;61:150-156.

27. Miguel EC, Coffrey BJ, Baer L, Savage CR, Rauch SL, Jenike MA. Phenomenology of intentional repetitive behaviors in obsessivecompulsive disorder and Tourette's disorder. J Clin Psychiatry 1995;56:246-255.

28. Cardoso F, Veado CCM, Oliveira JT. A Brazilian cohort of patients with Tourette's syndrome. J Neurol Neurosurg Psychiatry 1996;60:209-212.

29. Alves HL. Estudo epidemiológico e clínico dos tiques e da síndrome de Gilles de la Tourette em uma amostra da população de Indaiatuba SP. Dissertação de Mestrado. Faculdade de Ciências Médicas da Universidade Estadual de Campinas. Campinas, 1998. 